\title{
运行模式及参数对吸附制冷系统性能的影响*
}

\author{
朱龙潜 ${ }^{1}$ 吴池力 $^{2}$ 贺 伟 $^{1}$ 罗森文 $^{1}$ 胡韩莹 $^{3}$
}

(1. 广州市香港科大霍英东研究院 广州 510000;

2. 香港科技大学霍英东研究院 中国香港 999077 ;

3. 广州市万宝集团有限公司 广州 510000)

\begin{abstract}
摘要：因为气候变暖及能源短缺，新能源制冷技术受到了越来越多的关注。为了验证吸附制冷技术在实际应用中实现节能减 排的潜力及静电喷涂技术在吸附制冷系统中应用的可行性, 研发一台双箱吸附制冷系统。吸附剂由硅胶构成, 涂敷式吸附床 由静电喷涂技术喷涂而成。研发了一种新型回质回热运行模式, 为了探讨该运行模式对系统性能的影响, 设计、测试并对比 了多种运行模式, 发现添加了该新型运行模式具有一定的优越性, 相对于基本运行循环性能提升了 $113.7 \%$ 。试验中通过控制 变量法, 对多个运行参数进行了研究及优化, 例如: 吸附/脱附时间、回质时间、回热时间、冷却水进水温度、热水进水温度 等。发现各个运行参数对系统性能均有不同程度的影响。在 $25{ }^{\circ} \mathrm{C}$ 冷却水进水温度、90 ${ }^{\circ} \mathrm{C}$ 热水进水温度、16 ${ }^{\circ} \mathrm{C}$ 载冷水进水 温度、 $660 \mathrm{~s}$ 吸附/脱附时间、 $85 \mathrm{~s}$ 回质时间、 $50 \mathrm{~s}$ 回热时间、 $2 \mathrm{~kg} / \mathrm{min}$ 载冷水流速、 $8 \mathrm{~kg} / \mathrm{min}$ 热水以及冷却水流速的工况下, 系统能效比为 0.25 、单位制冷量为 $180.4 \mathrm{~W} / \mathrm{kg}$ 。由试验结果可见, 吸附制冷系统以及由静电喷涂技术制作而成吸附床对节能 减排有促进作用，有一定的应用前景。
\end{abstract}

关键词：吸附制冷系统; 运行模式; 运行参数; 静电喷涂; 系统性能

中图分类号: TB657

\section{Investigation of the Effect of Operating Sequences and Conditions on the Cooling Performance of Adsorption Cooling System}

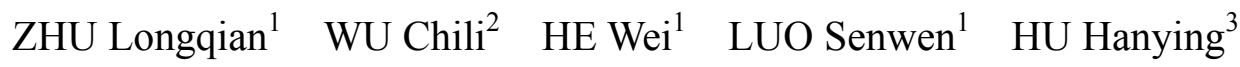 \\ (1. Guangzhou HKUST Fok Ying Tung Research Institute, Guangzhou 510000; \\ 2. Fok Ying Tung Graduate School, The Hong Kong University of Science and Technology, \\ Hong Kong 999077, China; \\ 3. Guangzhou Wanbao Group Company Limited, Guangzhou 510000)
}

\begin{abstract}
Because of the global warming and energy shortage, new energy cooling technologies have received more and more attention. In order to verify the potential of the adsorption cooling technology in the real application and the feasibility of the electrostatic coating method in an adsorption cooling system, a double-bed adsorption chiller was built. In this chiller, silica gel was used as the adsorbent, and the coated adsorbent beds were prepared with the electrostatic coating method. A novel heat and mass recovery phase was developed. In order to investigate its effect on the performance, various operating sequences were designed and tested. It was found that this novel operating sequence had a better performance than the basic operating sequence, which showed an improvement of $113.7 \%$. Various operating conditions for example, adsorption/desorption phase time, mass recovery time, heat recovery phase time, cooling water inlet temperature, and hot water inlet temperature, were investigated and optimized with the control variate method. It was found that the operating conditions had significant influence on the system performance. The coefficient of performance (COP) is recorded at about 0.25 , while the specific cooling power (SCP) is about $180.4 \mathrm{~W} / \mathrm{kg}$ under the
\end{abstract}

\footnotetext{
*广东省博士后(219600)、广州市科技(201807010097, 201907010036)和南海 科大专项资金(FSNH-18FYTRI01)资助项目。20200103 收到初稿, 20200902 收到修改稿
} 
operating condition of about $25{ }^{\circ} \mathrm{C}$ cooling water inlet temperature, $90{ }^{\circ} \mathrm{C}$ hot water inlet temperature, $16{ }^{\circ} \mathrm{C}$ chilled water inlet temperature, $660 \mathrm{~s}$ of adsorption/desorption phase time, $85 \mathrm{~s}$ of mass recovery time, $50 \mathrm{~s}$ of heat recovery time, $2 \mathrm{~kg} / \mathrm{min}$ chilled water flow rate, and $8 \mathrm{~kg} / \mathrm{min}$ hot water and cooling water flow rate. According to the experimental results, it was found that the adsorption chiller and the coated adsorbent bed had a positive impact on the energy-saving and a great potential on the real application.

Key words: adsorption chiller; operating sequence; operating condition; electrostatic coating; system performance

\section{0 前言}

在过去的几十年中, 全球气候变暖以及能源短 缺受到越来越多的关注。与此同时, 人们对电能的 需求(特别是在通风制冷设备上)越来越大, 而压缩 机空调是其中主要的耗电设备 ${ }^{[1]}$ 。由此可见, 若能 在空调设备上节约电能, 将能在节能减排这一目标 上做出巨大的贡献。在拥有废热、太阳能等低品位 热源的情况下，吸附式制冷是实现这一目标、替代 压缩式制冷、节省电能的一个可行方案。吸附制冷 系统主要由热能驱动, 可以采用水等无害物质作为 制冷剂(吸附质)来替代传统空调中的氟利昂等有害 物质, 因此吸附式制冷技术是一种节能环保的制冷 技术。吸附式制冷技术的研究从 20 世纪 70 年代开 始兴起, 然而当时并没有受到足够的重视。相对于 以前, 现在这项技术已经越来越成熟，同时也鼓励 了一部分用户在其房屋内安装试用了这种环保节能 的制冷系统 ${ }^{[2]}$ 。

吸附剂-吸附质工质对是吸附制冷系统的核心 之一。AHMED 等 ${ }^{[3]}$ 总结了一部分可用的工质对, 并把大部分吸附剂分类成了 3 种: 物理吸附剂、化 学吸附剂及复合吸附剂。硅胶是物理吸附剂, 而硅 胶-水是在吸附制冷系统中使用得比较普遍的工质 对。BOELMAN 等 ${ }^{[4]}$ 模拟及试验测试了一台以硅胶水作为工质对的吸附制冷系统。在 $50{ }^{\circ} \mathrm{C}$ 热水进水 温度及 $20{ }^{\circ} \mathrm{C}$ 冷却水进水温度的工况下, 系统能效 比(Coefficient of performance, COP)峰值测得为 0.4 。 WANG 等 ${ }^{[5]}$ 调研了 5 种适用于吸附制冷系统的工质 对, 分别为: 活性炭-水、活性炭-氨、分子篮-水、 硅胶-水及氯化钙-氨, 并认为硅胶-水最适合应用在 低温吸附制冷系统中。相对于其他工质对, 虽然硅 胶-水工质对的极限制冷量(Specific cooling power, $\mathrm{SCP}$ )及 COP 并不是最高的，但是有以下 2 个优势: 使用水作为吸附质, 环保无害; 硅胶需要的脱附温 度较低, 对热源要求较低, 适用性广。

除了工质对之外, 吸附床是吸附制冷系统中的 另一个核心。在吸附制冷系统的发展过程中, 有多 种吸附床被研发、研究及优化。ZHU 等 ${ }^{[6]}$ 研发了一
个新型吸附床, 由多个圆柱体换热器组成。圆柱体 换热器中有一条螺旋铜管作为传热管道, 外侧包裹 金属滤网, 吸附剂填充在滤网内。相对于车用换热 器, 这种新型的吸附器可以多填充 $40 \%$ 的吸附剂。 然而该填充式吸附床的传热传质速率过低，在试验 中 $\mathrm{COP}$ 为 $0.26, \mathrm{SCP}$ 仅为 $3.4 \mathrm{~W} / \mathrm{kg}$ 。 WANG 等 ${ }^{[7]}$ 研发了一种流体式吸附床并通过试验验证其效果。 流体式吸附床相对于传统的填充式吸附床, 能将吸 附质吸附脱附时的质量变化速率提高 $630 \%$ 。然而当 吸附剂超过 $3 \mathrm{~kg}$ 时, 其液化不完全，会大大降低其 效率。除了填充式吸附床及流体式吸附床, 涂敷式 吸附床也是一种主要的吸附床类型。TATLIER ${ }^{[8]}$ 研 发了以分子篎和金属有机框架为主体的涂敷式吸附 床。试验结果显示该吸附床的涂层最优厚度为 $130 \sim 170 \mu \mathrm{m}$ ，与此同时，应用了该吸附床的制冷系 统取得了 $80.2 \mathrm{~kW}$ 的制冷量。涂敷的技术多种多样, 其中静电喷涂技术是一种常用的涂敷技术，然而此 技术尚未在吸附制冷系统的研究中得到广泛的应用 及有效的验证。

在系统运行过程中，运行参数及运行循环模式 是影响系统性能的关键因素 ${ }^{[9]}$ 。MIYAZAKI 等 ${ }^{[10]}$ 研 发了一台蒸发式吸附制冷系统, 并研究了载冷水进 水温度及循环时间对系统性能的影响。由试验结果 得知, 载冷水进水温度越高, 系统 $\mathrm{SCP}$ 和 $\mathrm{COP}$ 越 高; 更长的预热预冷时间, 可以减小载冷水出水温 度的波动范围。TSO 等 ${ }^{[11]}$ 通过数值模拟研究了吸附 脱附时间、冷却水进水温度以及载冷水进水温度对 系统性能的影响。模拟结果表明吸附脱附时间、冷 却水进水温度对系统 SCP 和 $\mathrm{COP}$ 都有较大的影响, 而载冷水进水温度对 SCP 的影响最大。除模拟之外, $\mathrm{TSO}$ 等 ${ }^{[12]}$ 也通过试验验证了其模拟结果, 并建议了 一种回质阶段-预热预冷的循环模式。通过运行这种 循环模式, SCP 和 COP 分别比运用之前提升了 $129 \%$ 和 $100 \%$, 分别为 $106 \mathrm{~W} / \mathrm{kg}$ 和 0.16 。不同的运行参 数对不同的吸附制冷系统的影响不尽相同, 而运行 循环模式也有进一步提升的空间。

虽然现在已经有很多研究在改进吸附剂-吸附 质工质对、吸附器、运行模式等, 但是仍然有更多 的工作需要去完成。此次研究主要目的如下。 
(1) 研发一台双箱吸附制冷系统, 该系统使用 硅胶-水作为工质对, 采用静电喷涂技术制作其涂敷 式吸附床, 并由低温热源驱动并能产生 $5{ }^{\circ} \mathrm{C}$ 左右的 载冷水。

(2) 研发中一种新的运行循环模式, 并通过试 验验证这种新模式以及静电喷涂技术在吸附制冷系 统中应用的可行性。

(3) 通过试验研究不同的运行参数(包括热水进 水温度、冷却水进水温度、吸附脱附时间、回质时 间以及回热时间等)对系统性能的影响。

\section{1 吸附制冷系统工作原理}

相对于传统的压缩式制冷系统, 吸附制冷系统 没有压缩机, 使用太阳能、废热等低品位热源代替 电能来驱动。因此吸附制冷系统更省电, 从而使它 更具有吸引力。系统中有 4 个关键部件, 分别为: 吸附剂-吸附质工质对、吸附床、蒸发器以及冷凝器。

吸附制冷系统的工作原理为: 大量吸附剂(硅胶) 涂敷在 2 个吸附器中的吸附床上。2 个吸附器分别 为吸附箱和脱附箱。在吸附阶段, 在蒸发器中有大 量吸附质(水)并在低压下在低温沸腾吸热制冷, 并 产生气体气态吸附质(水汽); 吸附箱中的硅胶吸附 从蒸发器中传过来的水汽; 冷却水通入吸附箱, 通 过换热器带走吸附时产生的热量。经过一定时间后, 硅胶达到饱和。饱和的硅胶需要被加热再生, 此过 程为脱附阶段。热水通入脱附箱, 通过换热器加热 硅胶, 而水汽从硅胶中脱附。当脱附完全后, 硅胶 完成再生。由于冷凝器中气压比脱附箱气压低, 水 汽会流入冷凝器并冷凝成水珠。同时冷却水通入冷 凝器带走冷凝时产生的热量并保持冷凝器温度比脱 附的水汽温度低。水珠因为重力及压差作用, 流回 蒸发器。为了保证系统持续制冷, 需要 2 个吸附器, 一个吸附的同时另一个脱附, 并交叉循环。

一般吸附致冷系统的运行循环包括 2 个基本阶 段(阶段一和阶段二)。在阶段一中, 吸附器 $\mathrm{A}$ 为吸 附箱, 吸附器 $\mathrm{B}$ 为脱附箱; 在阶段二中, 吸附器 $\mathrm{A}$ 为脱附箱, 吸附器 $\mathrm{B}$ 为吸附箱。系统原理图如图 1 所示, 其中 V1、V2、V3、V4、V5 为真空阀, V6、 V7、V8、V9、V10、V11、V12、V13、V14、V15 为电动球阀, $\mathrm{P} 1 、 \mathrm{P} 2 、 \mathrm{P} 3 、 \mathrm{P} 4$ 为水葲。

在阶段一中, V1、V5、V6、V11、V13、V14 开启, 其他阀门则关闭。P1、P2、P4 运行而 P3 关 闭。水在低压蒸发器中快速蒸发, 水汽从蒸发器流 到吸附器 $\mathrm{A}$ 并被吸附剂吸附。冷却水从冷却塔流入
吸附器 $\mathrm{A}$, 并带走其中吸附热, 并流回冷却塔。此 时, 吸附器 $\mathrm{B}$ 被热水加热。水汽在高温下从吸附剂 中脱附, 流入冷凝器中并冷凝成水珠。水珠在冷凝 器中汇集成水流并流回蒸发器中。阶段二与阶段一 类似, 区别在于吸附器 $\mathrm{A}$ 为脱附箱而吸附器 $\mathrm{B}$ 为吸 附箱。因此, 在此阶段中, V2、V4、V7、V10、 $\mathrm{V} 12$ 和 V15 开启, 其他阀则关闭。P1、P2、P4 运 行而 P3 关闭。

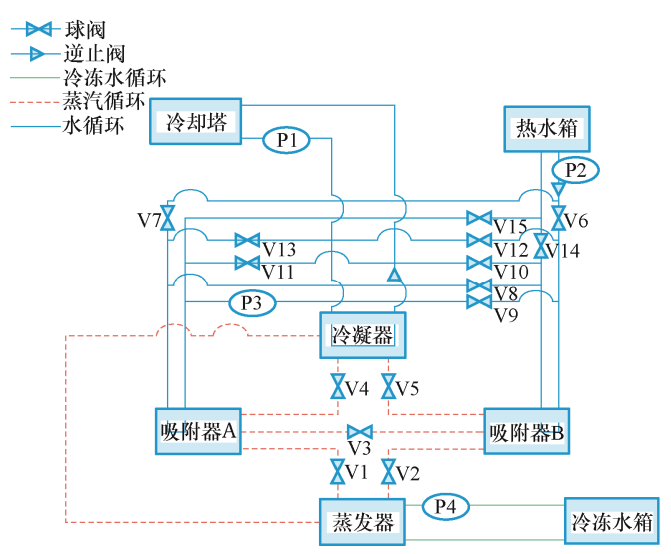

图 1 吸附制冷系统原理图

为了进一步提升系统性能, 在基本阶段之外, 还可以添加更多的运行阶段(例如: 回质阶段和回热 阶段)。本文研发了一种新型回质阶段及回热阶段, 应用在阶段一及阶段二之后, 其运行方式如下所述。

在阶段一完成之后, 吸附器 $\mathrm{B}$ 的气压远高于吸 附器 $\mathrm{A}$, 因此需要运行一个回质阶段(回质阶段一) 来平衡气压, 进一步帮助脱附。在回质阶段一中, 吸附器 $\mathrm{A}$ 和吸附器 $\mathrm{B}$ 连通, 同时冷却水持续流入吸 附器 $\mathrm{A}$ 而热水持续流入吸附器 $\mathrm{B}$ 。通过连接 2 个吸 附器, 水汽从高压的吸附器 $\mathrm{B}$ 中流入低压的吸附器 $\mathrm{A}$ 。在回质阶段一中, $\mathrm{V} 3 、 \mathrm{~V} 6 、 \mathrm{~V} 11 、 \mathrm{~V} 13$ 和 $\mathrm{V} 14$ 开启而其他都关闭; P1、P2 和 P4 运行而 P3 关闭。 在传统的回质阶段中, 并没有持续给 2 个吸附器注 入冷却水或热水, 因此吸附器 $\mathrm{B}$ 中的吸附剂只能在 温度持续降低的情况下通过气压降低来进一步脱 附。而在本文中的回质阶段中, 吸附器 $\mathrm{B}$ 中的吸附 剂因为能维持在高温, 所以能脱附出更多的水汽。 被脱附得更干燥的吸附剂在阶段二时, 能够吸附更 多的水汽。在阶段二完成之后, 同样需要运行一个 回质阶段 (回质阶段二)。在回质阶段二中, 吸附器 $\mathrm{A}$ 和吸附器 $\mathrm{B}$ 连通, 同时热水持续流入吸附器 $\mathrm{A}$ 而 冷却水持续流入吸附器 $\mathrm{B}$ 。V3、V7、V10、V12 和 $\mathrm{V} 15$ 开启而其他阀门关闭。P1、P2 和 P4 运行而 P3 关闭。回质阶段会持续一分钟左右, 完成之后需要 运行一个回热阶段。 
在回热阶段中, $\mathrm{V} 8$ 和 V9 开启而其他阀门关闭。 $\mathrm{P} 3$ 和 P4 运行而 P1 和 P2 关闭。吸附器 $\mathrm{A}$ 和吸附器 $\mathrm{B}$ 中的导热流体(冷却水、热水)连通, 因为温差, 热 能能被回收一部分。当 2 个吸附器中水温达到平衡 时，回热阶段完成。

在本文中, 完整的运行循环可以被描述为 “阶 段一 $\rightarrow$ 回质阶段一 $\rightarrow$ 回热阶段 $\rightarrow$ 阶段二 $\rightarrow$ 回质阶段 二 $\rightarrow$ 回热阶段 $\rightarrow$ 阶段一 $\rightarrow \cdots \cdots$

\section{2 动态方程及求解}

\section{1 吸附床详解}

吸附床是吸附制冷系统的关键部件之一。一个 好的吸附床设计意味着能够装载更多的吸附剂, 具 有更好的传质传热能力以及对导热流体具有更低的 摩擦阻力。由于硅胶的导热系数不高, 因此本文通 过静电喷涂技术将硅胶喷涂到吸附床上, 提高接触 面积, 从而提高其传质传热能力。板翅式换热器是 作为吸附床的比较好的选择, 因为它具有良好的传 热能力、廉价、组装简易以及方便进一步改进等优 点。在图 2 中, 展示了静电喷涂装置以及喷涂完成 之后的换热器。吸附剂喷涂在翅片上。换热器的尺 寸为 $270 \mathrm{~mm} \times 210 \mathrm{~mm} \times 45 \mathrm{~mm}$ 。

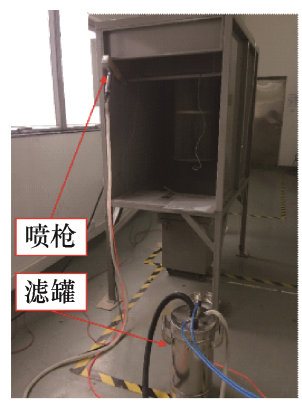

(a) 静电喷涂装置

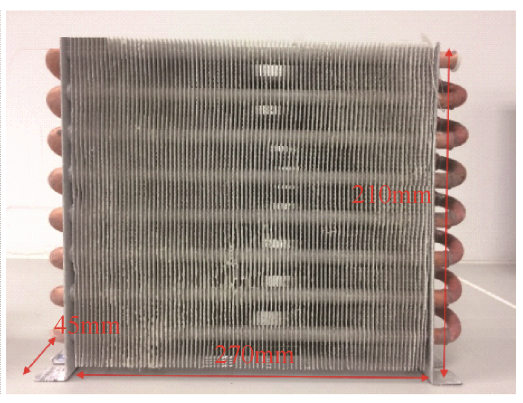

(b) 喷涂完成之后的换热器
图 2 静电喷涂装置及换热器

通过静电喷涂技术制作的涂敷式吸附床具体制 备过程如下。

(1) 对换热器表面进行油污去除及钝化。

(2) 将硅胶放入烘干机中进行干燥, 在 $150{ }^{\circ} \mathrm{C}$ 下烘干 $24 \mathrm{~h}$, 而后将干燥后的硅胶与粘合剂进行混 合(质量比为 $4: 1$ )并制粉, 将粉末装入图 2a 所示的 滤罐中。

（3）通过喷枪将滤罐中的粉末均匀喷涂在翅片 上(喷枪距离换热器 $20 \mathrm{~cm}$ 且喷枪与换热器垂直)。

(4) 将喷涂后的换热器置于烘干器中进行吸附 剂涂层固化, 在 $150{ }^{\circ} \mathrm{C}$ 下烘干 $24 \mathrm{~h}$ 。从烘干机取出 后即为完成涂敷的换热器。
静电喷涂技术具有以下优势。

(1) 吸附剂粉末均匀的喷涂在翅片上的各个部 分 $^{[13]}$ 。

（2）使用环氧聚酯作为粘合剂，不仅不会影 响吸附剂的性能, 还能将吸附剂紧紧地涂敷在翅 片上 上 $^{[14]}$ 。

（3）使用静电喷涂技术，可以有效地控制吸附 剂涂层的厚度 ${ }^{[15]}$, 且不会大幅增加热阻 ${ }^{[16]}$ 。

吸附床由 8 片换热器并联组成。每个换热器可 以喷涂 $0.7 \mathrm{~kg}$ 硅胶。在单个吸附器中, 总共可以喷 涂 $5.6 \mathrm{~kg}$ 吸附剂。涂层厚度约为 $0.5 \mathrm{~mm}$ 。吸附器上 装有热电偶及压力传感器, 用来监测吸附器内的温 度及压力。

图 3 分别展示了本文中使用的吸附制冷系统的 正面图及侧面图。如图 3 所示, 吸附制冷系统的主 要部件有: 冷凝器、吸附器、蒸发器、总控制器、 水泵以及热水箱。冷却塔则放置于室外并提供冷却 水给系统。

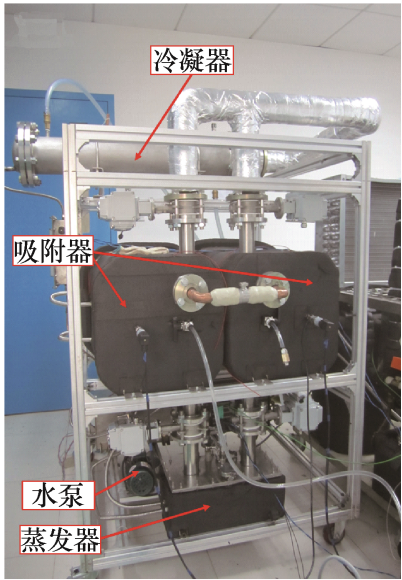

(a)正面图

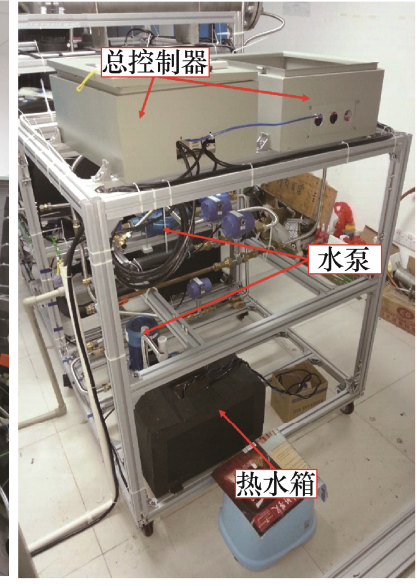

(b)侧面图
图 3 吸附制冷系统

\section{2 控制及辅助系统详解}

为了提供稳定的热源, 热水箱中安装了 $2 \mathrm{~kW}$ 及 $1 \mathrm{~kW}$ 的电加热器各一个; 为了提供稳定的冷却 水温度, 冷水塔中安装了一个 $2 \mathrm{~kW}$ 的电加热器, 并通过总控制器中的 PID 温度控制器进行控制。

所有由热电偶及压力传感器所得到的数据均由 数据采集设备所采集。其中, 热电偶信号由通过型 号为 SCXI-1102 的 32 通道热电偶/电压输入模块所 采集; 压力信号由型号为 USB-6229 的 USB 数据设 备采集并导入计算机中。在计算机中的数据采集界 面由 NI LabVIEW (Version 8.6)构建。数据每 $3 \mathrm{~s}$ 采 集一次并上传到计算机硬盘中。制冷系统中的真空 阀及球阀的开关通过型号为 ATmega128 的微控制 单元和继电器模块控制。水泵则通过无线远程控制 
模块进行控制。

\section{3 计算}

系统的 COP 由式(1)得出。需要注意的是, 该 公式算出的 $\mathrm{COP}$ 是平均值。

$$
\left.\mathrm{COP}=\int_{0}^{t_{\text {cycle }}}\left(Q_{\text {chill }} / Q_{\text {des }}\right) \mathrm{d} t\right) / \int_{0}^{t_{\text {cycle }}} \mathrm{d} t
$$

式中, $Q_{\text {chill }}$ 为制冷量; $Q_{\text {des }}$ 为消耗的热能; $t_{\text {cycle }}$ 为 完成一个完整循环需要的时间。系统的制冷量以及 热能能耗则通过式(2)和式(3)计算的出

$$
\begin{gathered}
Q_{\text {chill }}=\dot{m}_{\text {chill }} c_{p, \text { water }}\left(T_{\text {chill, in }}-T_{\text {chill, out }}\right) \\
Q_{\text {des }}=\dot{m}_{\text {hot }} c_{p, \text { water }}\left(T_{\text {hot , in }}-T_{\text {hot,out }}\right)
\end{gathered}
$$

式中, $\dot{m}_{c h i l l}$ 和 $\dot{m}_{h o t}$ 分别为载冷水流速和热水流速; $c_{p, \text { water }}$ 为水的比热容; $T_{\text {chill, in }}$ 和 $T_{\text {chill,out }}$ 分别为载冷水 进水温度及出水温度; $T_{h o t, \text { in }}$ 和 $T_{\text {hot }, \text { out }}$ 分别为热水进 水温度及出水温度。式(4)陈述了 $t_{\text {cycle }}$ 的计算方法

$$
t_{\text {cycle }}=2 t_{\text {ads }, \text { des }}+2 t_{m r}+2 t_{h r}
$$

式中, $t_{a d s, d e s}$ 为吸附/脱附时间; $t_{m r}$ 为回质时间; $t_{h r}$ 为回热时间。式(5)为 SCP 的计算方式

$$
S C P=Q_{\text {chill }} / W
$$

式中, $W$ 为单个吸附器中吸附剂的质量。

\section{3 试验结果及分析}

本文研究了不同的运行参数对系统的影响。 表 1 为吸附制冷系统的标准运行参数。由于热能能 耗是由热水箱的进出水温差计算得出, 因此热损失 也被计算在内。热损失主要包括从吸附器、热水箱、 管道散失到外界的能量。

表 1 标准运行参数

\begin{tabular}{lc}
\hline \multicolumn{1}{c}{ 参数 } & 数值 \\
\hline 脱附温度 $T_{d e s} /{ }^{\circ} \mathrm{C}$ & 90 \\
冷却水进水温度 $T_{c \text { cool, in }} /{ }^{\circ} \mathrm{C}$ & 25 \\
载冷水进水温度 $T_{c h i l, i n} /{ }^{\circ} \mathrm{C}$ & 16 \\
吸附/脱附时间 $t_{a d s, d e s} / \mathrm{s}$ & 600 \\
回质时间 $t_{m r} / \mathrm{s}$ & 75 \\
回热时间 $t_{h r} / \mathrm{s}$ & 55 \\
热水流速 $\dot{m}_{h o t} /(\mathrm{kg} / \mathrm{min})$ & 8 \\
冷却水流速 $\dot{m}_{c o o l} /(\mathrm{kg} / \mathrm{min})$ & 8 \\
载冷水流速 $\dot{m}_{c h i l l} /(\mathrm{kg} / \mathrm{min})$ & 2 \\
\hline
\end{tabular}

除了标准运行参数, 本文通过控制变量法, 测 试各项运行参数对系统性能的影响。具体测试的参 数及范围如表 2 所示。由于吸附制冷系统可以用廉 价甚至是免费的低品位热源(例如: 太阳能, 废热等)
驱动, 因此 SCP 是描述系统性能的最主要参数, 而 $\mathrm{COP}$ 仅作为参考作用。在优化参数的试验中, 最佳 参数的判定以 SCP 的高低作为主要依据, 以 $\mathrm{COP}$ 的高低为次要依据。

表 2 多项不同的运行参数

\begin{tabular}{ll}
\hline \multicolumn{1}{c}{ 参数 } & \multicolumn{1}{c}{ 数值 } \\
\hline 热水进水温度 $T_{d e s} /{ }^{\circ} \mathrm{C}$ & $55,65,75,85$ \\
冷却水进水温度 $T_{c o o l, i n} /{ }^{\circ} \mathrm{C}$ & 15,35 \\
吸附/脱附时间 $t_{a d s, d e s} /{ }^{\circ} \mathrm{C}$ & $480,540,660,720$ \\
回质时间 $t_{m r} / \mathrm{s}$ & $55,65,85,95$ \\
回热时间 $t_{h r} / \mathrm{s}$ & $45,50,60,65,70,75$ \\
\hline
\end{tabular}

\section{1 冷却水进水温度对系统性能的影响}

冷却水进水温度对系统性能的影响的试验结果 如图 4 所示。由图 4 可见, 当冷却水温度升高时, 系统性能会有所降低。系统在 $35{ }^{\circ} \mathrm{C}$ 冷却水进水温 度时的 $\mathrm{SCP}$ 和 $\mathrm{COP}$ 相较于 $15{ }^{\circ} \mathrm{C}$ 冷却水进水温度时 分别降低了 $9 \%$ 和 $4.3 \%$ 。当冷却水进水温度更低时, 吸附剂可以在吸附阶段吸附更多的水汽，从而使 $\mathrm{SCP}$ 和 COP 更高。即使冷却水进水温度高达 $35{ }^{\circ} \mathrm{C}$, 该系统仍然保持着稳定的制冷效果。由于 $25{ }^{\circ} \mathrm{C}$ 的 冷却水温度更贴近系统实际运行的温度, 因此冷却 水进水温度被设置为 $25{ }^{\circ} \mathrm{C}$ 。

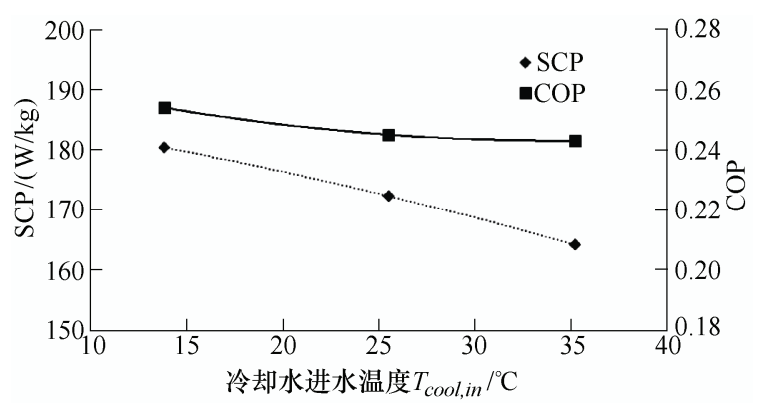

图 4 冷却水进水温度对系统性能的影响

\section{2 吸附/脱附时间对系统性能的影响}

图 5 所展示的是吸附/脱附时间对系统性能的影 响的试验结果。从图 5 中可见, SCP 和 COP 的最大 值均出现在吸附/脱附时间为 $660 \mathrm{~s}$ 的时候。当吸附/ 脱附时间过短时, 吸附箱中的吸附剂不能完全达到 饱和而脱附箱中的吸附剂不能完全再生, 因此导致 了吸附剂利用不完全, 系统性能的降低。当吸附/ 脱附时间过长时, 吸附剂达到饱和后, 不能吸附更 多的水汽，从而使气压持续上升, $\mathrm{SCP}$ 持续下降。 在实际运行过程中, 当吸附/脱附时间过长时, 制冷 量持续降低, 然而热损基本维持不变, 因此 COP 会 持续降低。在这种情况下, $660 \mathrm{~s}$ 为本文中的吸附制 冷系统的最优吸附/脱附时间。 


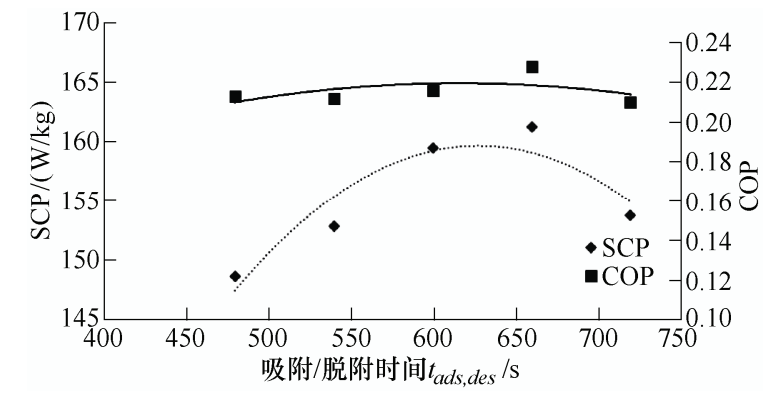

图 5 吸附/脱附时间对系统性能的影响

\section{3 回质时间及回热时间对系统性能的影响}

图 6 展示了回质时间对系统性能的影响。SCP 的最大值出现在 $85 \mathrm{~s}$, 而 COP 的最大值出现在 $75 \mathrm{~s}$ 。 在回质阶段中, 水汽因为压差从高压的脱附箱流入 到低压的吸附箱, 直到 2 个吸附器中的气压平衡为 止。由于在回质阶段中持续给脱附箱提高热水, 因 此系统在持续消耗热能。然而在回质阶段, 2 个吸 附器与蒸发器断开, 因此系统此时基本没有制冷量。 所以回质时间不能过长。然而过短的回质时间, 会 导致回质不完全, 也不一定能带来更好的制冷效果。 在本文中, $85 \mathrm{~s}$ 是系统的最优回质时间。

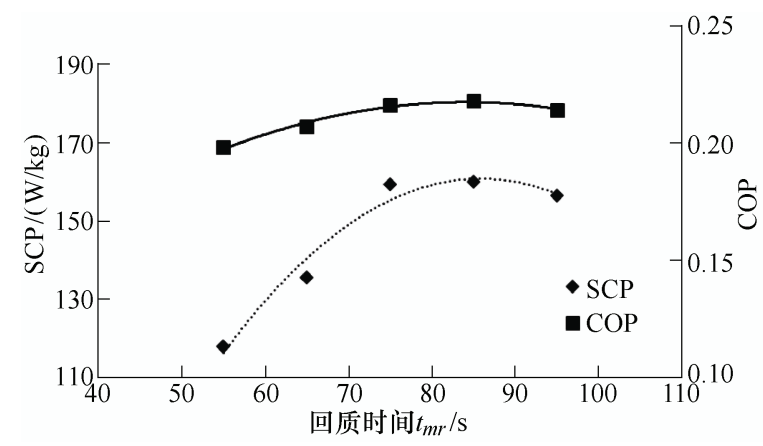

图 6 回质时间对系统性能的影响

图 7 所展示的是回热时间对系统 SCP 和 COP 的影响。SCP 在 $50 \mathrm{~s}$ 时达到最大值, 但 COP 在 $65 \mathrm{~s}$ 时达到最大值。运行回热阶段可以使系统在下一个 循环的实时制冷量更快地达到最高值。与回质阶段 类似, 在回热阶段时, 系统基本没有制冷效果, 因 此回热时间不能过长。然而过短的回热时间会导致 回热不完全, 同样不一定能带来更好的制冷效果。 在本文中, $50 \mathrm{~s}$ 为系统的最优回热时间。

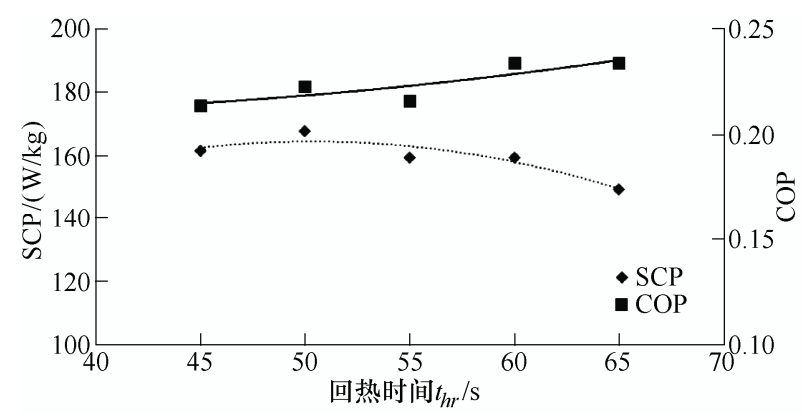

图 7 回热时间对系统性能的影响

\section{4 热水进水温度对系统性能的影响}

本文中, 通过控制热水箱中的电加热器, 分别 给吸附制冷系统提供了 5 组不同的热水进水温度。

图 8 为热水进水温度对系统性能的影响。在吸附制 冷系统中, 热水进水温度是对系统制冷效果, 特别 是 SCP, 影响最大的一个参数。相对于 $55{ }^{\circ} \mathrm{C}$ 热水 进水温度时, 系统在 $90{ }^{\circ} \mathrm{C}$ 热水进水温度时的 $\mathrm{SCP}$ 提升了 $207 \%$ 。更高的热水进水温度可以使吸附剂在 脱附时达到更高的温度, 从而脱附出更多的水汽。 因此在下一个循环时，可以吸附更多的水汽，从而 增大系统的制冷量。然而在本文的吸附制冷系统中, 热水进水温度对 COP 的影响并不是很大, $\mathrm{COP}$ 的 最大值和最小值的差距仅为 $5 \%$ 。COP 在 $90{ }^{\circ} \mathrm{C}$ 时相 对于 $85{ }^{\circ} \mathrm{C}$ 有略微的下降, 主要原因则是热损在高 温时有略微的增大。

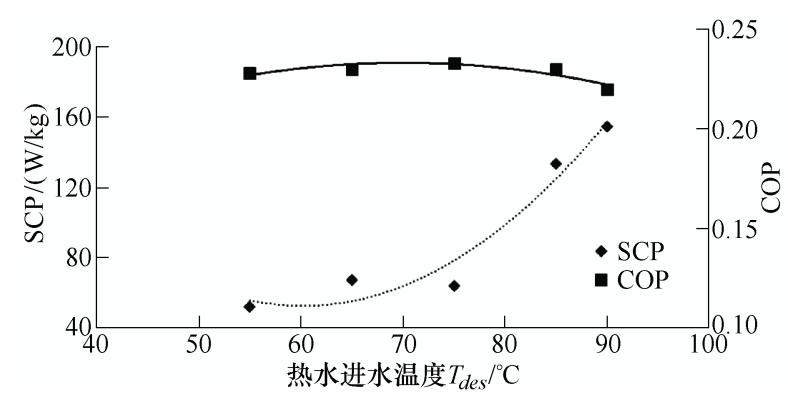

图 8 热水进水温度对系统性能的影响

本文对吸附制冷系统的多个运行参数的最优值 进行了研究, 然而最优值具体的数值取决于多个原 因, 例如: 吸附剂总重量、吸附床及吸附器的设计、 管道的设计、阀门的大小等。因此, 系统一旦有所 改变或改进, 最优值也会相应的进行变化。

\section{5 运行模式对系统性能的影响}

本文采用了新型的回质阶段而非传统的回质 阶段, 因此有必要研究这种新型的回质阶段以及 回热阶段对系统性能的影响。如表 3 中所示, 本 文总共测试了 7 种不同的运行模式。其中，基本 循环可以被描述为 “阶段一 $\rightarrow$ 阶段二 $\rightarrow$ 阶段一 $\rightarrow \cdots$ ”。基本循环+传统回质阶段可以被描述为“阶 段一 $\rightarrow$ 传统回质阶段 $\rightarrow$ 阶段二 $\rightarrow$ 传统回质阶段 $\rightarrow$ 阶 段一 $\rightarrow \cdots$ ”。基本循环+回热阶段可以被描述为 “阶 段一 $\rightarrow$ 回热阶段 $\rightarrow$ 阶段二 $\rightarrow$ 回热阶段 $\rightarrow$ 阶段一 $\rightarrow \cdots ”$ 。基本循环 + 传统回质阶段+回热阶段可以被 描述为 “阶段一 $\rightarrow$ 传统回质阶段 $\rightarrow$ 回热阶段 $\rightarrow$ 阶段 二 $\rightarrow$ 传统回质阶段 $\rightarrow$ 回热阶段 $\rightarrow$ 阶段一 $\rightarrow \cdots$ ”。基 本循环+本文中的回质阶段可以被描述为“阶段一 $\rightarrow$ 回质阶段一 $\rightarrow$ 阶段二 $\rightarrow$ 回质阶段二 $\rightarrow$ 阶段一 $\rightarrow \cdots ”$ 。基本循环+回质阶段+回热阶段则为前文章 
节 1 中所描述的完整运行循环。

表 3 多种不同的运行模式及试验结果

\begin{tabular}{lcc}
\hline 运行模式 & $\mathrm{SCP} /(\mathrm{W} / \mathrm{kg})$ & $\mathrm{COP}$ \\
\hline 基本循环 & 84.4 & 0.13 \\
基本循环+传统回质(15 s) & 99.2 & 0.21 \\
基本循环+回热(50 s) & 90.6 & 0.23 \\
基本循环+传统回质(15 s)+回热(50 s) & 105.4 & 0.28 \\
基本循环+回质(15 s) & 122.4 & 0.19 \\
基本循环+回质(15 s)+回热(50 s) & 130.2 & 0.21 \\
基本循环+回质 $(85 \mathrm{~s})+$ 回热(50 s) & 180.4 & 0.25 \\
\hline
\end{tabular}

在本文中, 系统的传统回质阶段的最优回质时 间为 $15 \mathrm{~s}$, 因此在运行模式的对比试验中, 传统回 质阶段时间为 $15 \mathrm{~s}$, 而本文使用的新型回质阶段则 有 2 组, 分别为 $15 \mathrm{~s}$ 及 $85 \mathrm{~s}$ 。与基本循环相比, 使 用了传统回质阶段的循环的 SCP 提升了 $17.5 \%$ 。当 使用与传统回质阶段时间长短一致的新型回质阶段 时 $(15 \mathrm{~s}), \mathrm{SCP}$ 比基本循环提高了 $54.3 \%$ 。当回质阶 段时间达到最优时 $(85 \mathrm{~s})$, SCP 提升高达 $113.7 \%$ 。 由试验结果可见, 回质阶段, 特别是新型回质阶段
对系统性能有巨大的影响。回热阶段则对系统的 COP 影响较大。

\section{6 导热流体在各个位置的温度曲线}

图 9 所展示的是在优化后的运行参数下, 导热 流体在各个位置的温度曲线。系统的最优运行参数 如下: $90{ }^{\circ} \mathrm{C}$ 热水进水温度、 $25{ }^{\circ} \mathrm{C}$ 冷却水进水温度、 $16{ }^{\circ} \mathrm{C}$ 载冷水水进水温度、 $660 \mathrm{~s}$ 吸附/脱附时间、 $85 \mathrm{~s}$ 回质时间、 $50 \mathrm{~s}$ 回热时间、 $8 \mathrm{~kg} / \mathrm{min}$ 热水/冷却水流 速以及 $2 \mathrm{~kg} / \mathrm{min}$ 载冷水流速。载冷水出水温度在运 行过程中一直低于载冷水入水温度, 证明了系统有 稳定的持续制冷能力。在试验中, 载冷水出水温度 为 $5 \sim 7{ }^{\circ} \mathrm{C}$, 符合预定目标。热水进水温度在完成 回热阶段之后有个快速的、较大幅度的下降, 这表 示了有一部分完成了回热阶段之后的低温水涌入了 热水箱并导致了热水温度下降, 同时也表明了由静 电喷涂技术制作的涂敷式吸附床具有良好的传热能 力。热水与冷却水流速相近, 然而热水温度在完成 回热阶段之后下降幅度大, 而冷却水温度下降幅度 小, 是因为冷却塔容量为 $200 \mathrm{~L}$ 而热水箱容量仅为 $50 \mathrm{~L}$ 。

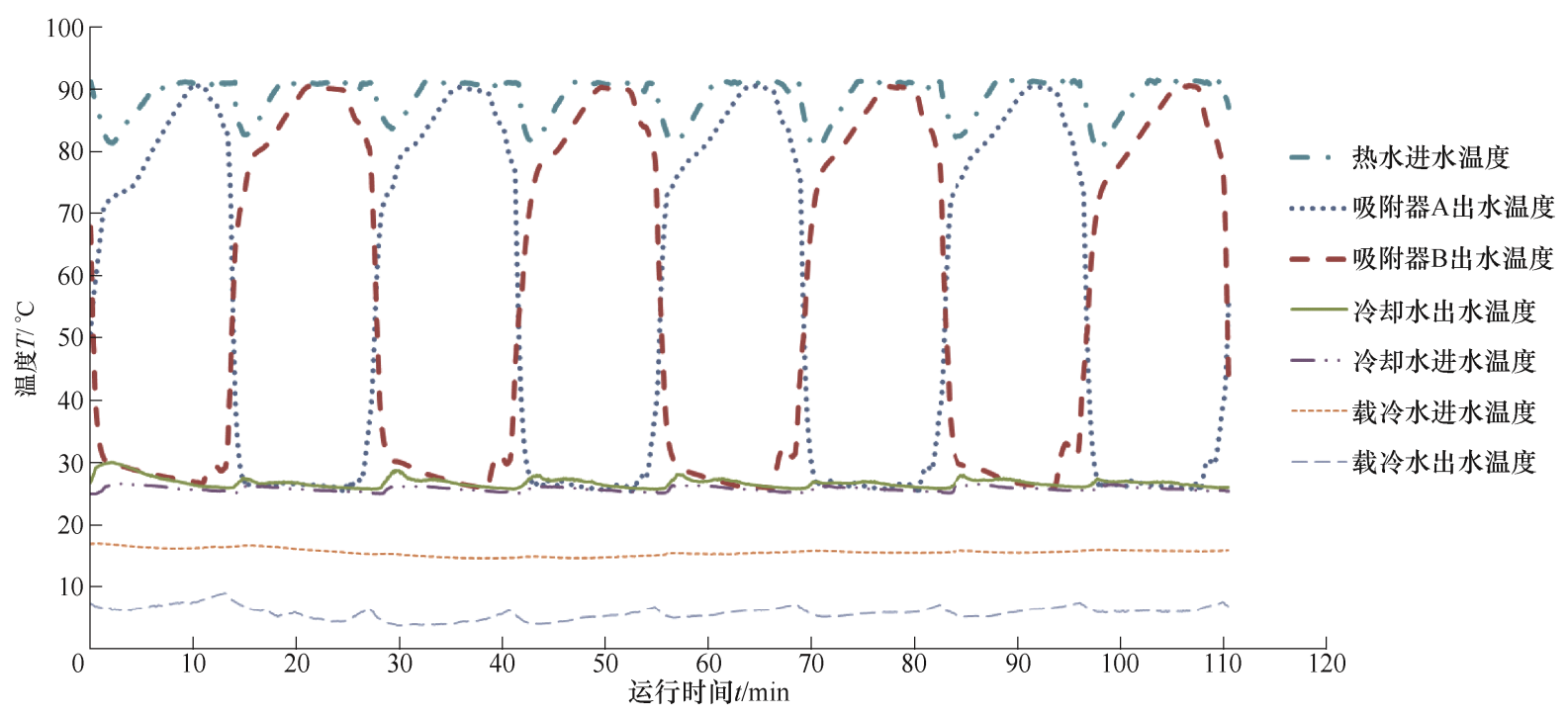

图 9 导热流体在各个位置的温度曲线

图 10 所展示的是吸附制冷系统的实时制冷 量。实时制冷量在一个循环开始 $3 \mathrm{~min}$ 左右达到最 高点, 然后开始缓慢下降。在前 $3 \mathrm{~min}$ 内, 实时制 冷量的提升是因为循环刚开始时, 吸附剂最干燥, 能吸附大量的水汽, 从而使得气压维持在一个较 低的水平 $(500 \mathrm{~Pa}$ 左右 $)$ 。在循环刚开始时, 吸附 剂的温度为 $60{ }^{\circ} \mathrm{C}$ 左右, 并随着系统运行, 冷却 水的流入而逐渐降低。吸附剂温度的降低可以增 大吸附剂的吸附量, 同样也是实时制冷量在前期 能提升的原因。3 分钟之后, 大部分在表面的吸
附剂已经饱和, 温度已经稳定下来之后, 水汽被 吸附变得困难, 吸附剂的吸附速率开始下降, 然 而蒸发器中的水还在持续蒸发, 于是气压开始上 升, 从而使水的沸点升高, 实时制冷开始降低。 在回质阶段以及回热阶段中, 2 个吸附器都与蒸 发器断开, 蒸发器气压会进一步升高, 因此实时 制冷量会进一步降低。然而, 回质阶段和回热阶 段依然是有必要的。在回质阶段和回热阶段中, 能使吸附剂更干燥, 并在下一个循环中吸附更多 的水汽，从而提高实时制冷量的最大值。 


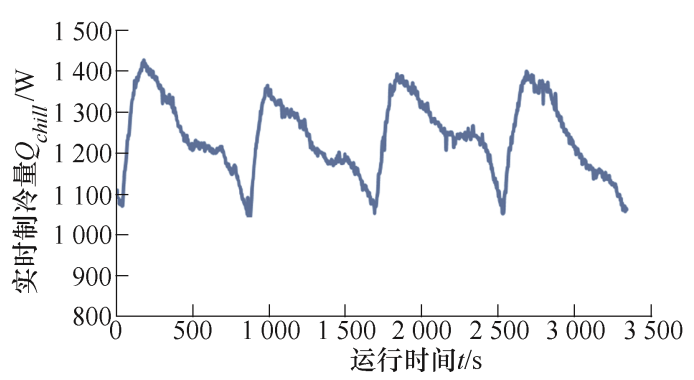

图 10 吸附制冷系统实时制冷量

\section{4 结论}

在本文中, 研发了一台吸附制冷系统。吸附器 中的吸附床由 8 个换热器组成, 并通过静电喷涂技 术涂敷了 $5.6 \mathrm{~kg}$ 吸附剂。系统中使用了硅胶-水作为 吸附剂-吸附质工质对。本文主要研究了不同的运行 参数、运行模式对系统性能的影响以及对一种新型
的回质阶段进行了研究及分析。

在标准运行参数下, 系统的 SCP 为 $159.5 \mathrm{~W} / \mathrm{kg}$, COP 为 0.21 。由参数优化试验的结果可见, 在最优 的运行参数下, 系统 SCP 提高到了 $180.4 \mathrm{~W} / \mathrm{kg}, \mathrm{COP}$ 提高到了 0.25 , 分别提升了 $13.1 \%$ 及 $17.6 \%$ 。

表 4 中对本文中的吸附制冷系统与其他类似 的研究的性能进行了罗列。此表并非为了比较, 而是希望对吸附制冷系统的研究有更全面的认识 及了解。在 FRENI 等 ${ }^{[24]}$ 的研究中, 研发了一种由 硅胶和硝酸钙组成的新型的复合吸附剂 $(\mathrm{SWS}-8 \mathrm{~L})$ )。在 $90{ }^{\circ} \mathrm{C}$ 热水进水温度, $15{ }^{\circ} \mathrm{C}$ 载冷水 进水温度, $30{ }^{\circ} \mathrm{C}$ 冷却水进水温度以及 30 分钟循 环时间的工况下, 其 SCP 高达 $389 \mathrm{~W} / \mathrm{kg}$ 。在 LU 等 ${ }^{[25]}$ 的研究中, 研发了一台一体化的吸附制冷系 统。其蒸发器, 吸附器及冷凝器集成在一起。在 试验中, 其 $\mathrm{SCP}$ 为 $153 \mathrm{~W} / \mathrm{kg}$ 。

表 4 本文吸附制冷系统的 SCP 与其他相似研究的系统的 SCP 的对比

\begin{tabular}{|c|c|c|c|c|c|c|}
\hline 研究 & 吸附床类型 & 工质对 & $\begin{array}{c}\text { 脱附温度 } \\
T_{d e s}{ }^{\circ} \mathrm{C} \\
\end{array}$ & $\begin{array}{c}\text { 载冷水进水温度 } \\
T_{\text {chill,in }} /{ }^{\circ} \mathrm{C} \\
\end{array}$ & $\begin{array}{c}\text { 循环时间 } \\
t_{c y c l e} / \mathrm{s} \\
\end{array}$ & $\begin{array}{c}\mathrm{SCP} / \\
(\mathrm{W} / \mathrm{kg}) \\
\end{array}$ \\
\hline 本文 & 涂敷式 & 硅胶-水 & 95 & 15.7 & 1590 & 180.4 \\
\hline WANG 等[17] & 填装式 & 硅胶-水 & 85 & 14 & 2280 & 63.4 \\
\hline LIU 等[18] & 填装式 & 硅胶-水 & 85 & 14 & 1020 & 52.8 \\
\hline WANG 等[19] & 填装式 & 硅胶-水 & 60 & 20.5 & 2280 & 46.2 \\
\hline CHAN 等[20] & 涂敷式 & 硅胶-水 & 80 & 14 & 720 & 176 \\
\hline XIA 等[21] & 填装式 & 硅胶-水 & 84 & 21.5 & 1920 & 104.6 \\
\hline $\mathrm{CHAN}$ 等 ${ }^{[22]}$ & 填装式 & 硅胶-水 & 80 & 14 & 1560 & 72 \\
\hline $\mathrm{CHEN}$ 等[23] & 填装式 & 硅胶-水 & 85 & 11.7 & 1680 & 132 \\
\hline FRENI 等 $^{[24]}$ & 涂敷式 & SWS-8L-水 & 90 & 15 & 600 & 389 \\
\hline LU 等[25] & 填装式 & 硅胶-水 & 86 & 20 & 720 & 153 \\
\hline WANG 等 ${ }^{[26]}$ & 填装式 & 硅胶-水 & 85 & 12.2 & 336 & 140 \\
\hline
\end{tabular}

总而言之, 通过本文的研究发现, 回质阶段(特 别是本文提出的新型回质阶段)、回热阶段、运行参 数对系统性能有明显的影响; 而板翅式换热器因其 廉价, 换热性好, 组装简易, 在用作吸附床时具有 一定的优势; 与此同时, 相对于填装式吸附床, 通 过静电喷涂技术处理的喷涂吸附床具有更好的传热 效率, 对系统的 SCP 有积极的影响, 在吸附制冷技 术中具有应用前景。

\section{参 考 文 献}

[1] Electrical \& Mechanical Services Department. Hong Kong energy end-use data 2013[R]. HK: Electrical \&
Mechanical Services Department, 2013.

[2] SAHA B B, KOYAMA S, KASHIWAGI T, et al. Waste heat driven dual-mode , multi-stage , multi-bed regenerative adsorption system[J]. International Journal of Refrigeration, 2003, 26: 749-757.

[3] AHMED A, SALEM M, ISNAEL I M, et al. An overview on adsorption pairs for cooling[J]. Renewable \& Sustainable Energy Reviews, 2012， 19: 565-572.

[4] BOELMAN E C, SAHA B B, KASHIWAGI T. Experimental investigation of a silica gel-water adsorption refrigeration cycle: The influence of operating conditions on cooling output and COP $[J]$. ASHRAE 
Transactions: Research, 1995, 101: 358-366.

[5] WANG Dechang, LI Yanhui, LI Da, et al. A review on adsorption refrigeration technology and adsorption deterioration in physical adsorption systems[J]. Renewable \& Sustainable Energy Reviews, 2009, 14: 344-353.

[6] ZHU Longqian, WU Chili, CHAN T C, et al. Development of a new multi-chamber double-layer adsorber in energy efficient solar adsorption cooling systems $[\mathrm{C}] / /$ Proceedings of the APEC Conference on Low Carbon Town and Physical Energy Storage. Changsha, China, 2013, E02-15: 125-130.

[7] WANG Q, GAO X, XU J Y, et al. Experimental investigation on a fluidized-bed adsorber/desorber for the adsorption refrigeration system[J]. International Journal of Refrigeration, 2012，35: 694-700.

[8] TARLIER M. Performances of MOF vs. zeolite coatings in adsorption cooling applications[J]. Applied Thermal Engineering, 2017, 113: 290-297.

[9] SAPIENZA A, SANTAMARIA S, FRAZZIC A, et al. Influence of the management strategy and operating conditions on the performance of an adsorption chiller[J]. Energy, 2011, 36: 5532-5538.

[10] MIYAZAKI T, AKISAWA A, SAHA B B. The performance analysis of a novel dual evaporator type three-bed adsorption chiller[J]. International Journal of Refrigeration, 2010, 33: 276-285.

[11] TSO C Y, CHAO C Y H, FU S C. Performance analysis of a waste heat driven activated carbon based composite adsorbent-water adsorption chiller using simulation model[J]. International Journal of Heat and Mass Transfer, 2012, 55: 7596-7610.

[12] TSO C Y, CHAN K C, CHAO C Y H, et al. Experimental performance analysis on an adsorption cooling system using zeolite $13 \mathrm{X} / \mathrm{CaCl}_{2}$ adsorbent with various operation sequences[J]. International Journal of Heat and Mass Transfer, 2015, 85: 343-355.

[13] YANG Qingliang, MA Yingliang, ZHU J, et al. An update on electrostatic powder coating for pharmaceuticals[J]. Particuology, 2017, 31: 1-7.

[14] 方玉堂, 刘灿, 张正国, 等. 管套翅片换热器吸附 剂涂层的制备方法: 中国, CN102601024A [P]. 2012-07-25.
FANG Yutang, LIU Can, ZHANG Zhengguo, et al. The preparation method of an adsorbent coating on a finned-tube heat exchanger: China, CN102601024A [P]. 2012-07-25.

[15] LUO Yanfeng, ZHU J, MA Yingliang, et al. Dry coating, a novel coating technology for solid pharmaceutical dosage forms[J]. International Journal of Pharmaceutics, 2008, 358: 16-22.

[16] RESTUCCIA G , CACCIOLA G. Performance of adsorption systems for ambient heating and air conditioning $[\mathrm{J}]$. International Journal of Refrigeration, 1999, 22(1): 18-26.

[17] WANG Dechang, WU Jingyi, XIA Zaizhong, et al. Study of a novel silica gel-water adsorption chiller, part II experimental study[J]. International Journal of Refrigeration, 2005, 28: 1084-1091.

[18] LIU Yanling, WANG Ruzhu, XIA Zaizhong. Experimental performance of a silica gel-water adsorption chiller[J]. Applied Thermal Engineering, 2005, 25 : 359-375.

[19] WANG Ruzhu, OLIVEIRA R G. Adsorption refrigeration: An efficient way to make good use of waste heat and solar energy[J]. Progress in Energy and Combustion Science, 2006, 32: 424-458.

[20] CHANG W S, WANG C C, SHIEH C C. Experimental study of a solid adsorption cooling system using flat-tube heat exchangers as adsorbent bed[J]. Applied Thermal Engineering, 2007, 27: 2195-2199.

[21] XIA Zaizhong, WANG Dechang, ZHANG Jincui. Experimental study on improved two-bed silica gel-water adsorption chiller[J]. Energy Conversion Management, 2008, 49: 1469-1479.

[22] CHANG W S, WANG C C, SHIEH C C. Design and performance of a solar-powered heating and cooling system using silica-gel/water adsorption chiller[J]. Applied Thermal Engineering, 2009, 29: 2100-2105.

[23] CHEN Chuanjuan, WANG Ruzhu, XIA Zaizhong, et al. Study on a compact silica gel-water adsorption chiller without vacuum valves: Design and experimental study[J]. Applied Energy, 2010， 87: 2673-2681.

[24] FRENI A, SAPIENZA A, GLAZNEV I S, et al. Experimental testing of a lab-scale adsorption chiller 
using a novel selective water sorbent "silica modified by calcium nitrate" $[\mathrm{J}]$. International Journal of Refrigeration, 2012, 35: 518-524.

[25] LU Zisheng, WANG Ruzhu, XIA Zaizhong. Experimental analysis of an adsorption air conditioning with micro-porous silica gel-water[J]. Applied Thermal Engineering, 2013, 50: 1015-1020.

[26] WANG Xiaolin, HE Zhilong, CHUA H T. Performance simulation of multi-bed silica gel-water adsorption chillers[J]. International Journal of Refrigeration, 2015, 52: $32-41$

作者简介: 朱龙潜, 男, 1988 年出生, 博士, 研究助理教授。主要研究 方向为吸附制冷、冷凝、纳米涂层等。

E-mail: lzhuac@connect.ust.hk

吴池力 (通信作者), 男, 1984 年出生, 博士, 研究助理教授, 硕士研究 生导师。主要研究方向为室内空气质量、吸附制冷、小型风力发电和电 池热管理等。

E-mail: chiliwu@ust.hk 